\title{
Dissociation of Shaft of Humerus from Head in Three- part Fracture Dislocation due to Stress Concentration at Intramedullary Nail End
}

\author{
${ }^{1}$ Devendra Chouhan, ${ }^{2}$ Vishal Kumar, ${ }^{3}$ Manish Kundanmal Kothari, ${ }^{4}$ Mandeep S Dhillon
}

\begin{abstract}
A three-part fracture dislocation of the proximal humerus usually dissociates from the shaft at the level of the surgical neck or the anatomical neck. Dissociation from the shaft below this level has not reported in the literature before. Here we describe the injury of a middle aged patient with a three-part fracture dislocation of the humerus with dissociation of the head from the shaft at the level of proximal shaft humerus with a 25 years old Rush nail in situ. The dislocated head was found abutting the thoracic wall. This case report highlights the effect of stress concentration at intramedullary nail ends in the upper limb as well as the need for an extended approach when the dislocated head appears close to the thoracic wall.
\end{abstract}

Keywords: Shoulder fracture, Intramedullary fracture fixation, Mechanical stress.

How to cite this article: Chouhan D, Kumar V, Kothari MK, Dhillon MS. Dissociation of Shaft of Humerus from Head in Three-part Fracture Dislocation due to Stress Concentration at Intramedullary Nail End. J Postgrad Med Edu Res 2015;49(1): 46-48.

\section{Source of support: Nil}

Conflict of interest: None

\section{INTRODUCTION}

Proximal humerus fractures are relatively common in the elderly osteopenic patients. More than $85 \%$ of these fractures are only minimally displaced. A threepart fracture dislocation most commonly involves the surgical or anatomical neck and greater tuberosity. ${ }^{1}$ A three-part fracture dislocation with fracture involving the proximal shaft of humreus instead of the usual neck of humerus, has not been reported in literature in the

\footnotetext{
${ }^{1,2}$ Assistant Professor, ${ }^{3}$ Fellow, ${ }^{4}$ Professor and Head

1,2,4 Department of Orthopedics and Physical Medicine, Postgraduate Institute of Medical Education and Research, Chandigarh India

${ }^{3}$ Department of Spine, PD Hinduja National Hospital and Medical Research Centre, Mumbai, Maharashtra, India

Corresponding Author: Manish Kundanmal Kothari, Fellow Department of Spine, PD Hinduja National Hospital and Medical Research Centre, Mumbai, Maharashtra, India, Phone: 02224447108, e-mail: manishkkothari@gmail.com
}

authors best knowledge. We report the first such case of three-part fracture dislocation involving the greater tuberosity and proximal shaft of humerus with a 25 years old Rush nail in situ.

\section{CASE REPORT}

A 60-year-old male presented in the emergency department with acute pain in right shoulder following road traffic accident 8 hours ago. He was travelling on a bike when a car hit him from the side. He was violently thrown off his motorcycle followed by fall on an outstretched hand. He had no other complaints. His general condition was fair with fully awake, conscious and oriented. On local examination, the patient held his right upper limb in the opposite hand in adduction. There was a bruise on anteromedial aspect of his right shoulder. He also had an old scar on lower arm posteriorly. There was generalized swelling all around the shoulder as compared to the opposite side. The shoulder was tender to palpation. Due to the swelling, no clinical signs of dislocated shoulder were apparent. There was abnormal mobility palpable in proximal humerus. He did not have any numbness of proximal lateral arm. There was no distal neurovascular deficit. He was a known diabetic on erratic oral hypoglycemic and hypertensive. He gave history of fracture of his right arm bone for which he underwent surgery 25 years ago. No records were available with the patient. Radiographs (Fig. 1) of the right shoulder revealed a threepart fracture dislocation of proximal humerus.

Also there was an intramedullary Rush nail with healed fracture of lower shaft of humerus. The proximal humeral fracture involved the greater tuberosity and a long spiral fracture of proximal humeral shaft. About $2 \mathrm{~cm}$ of the proximal tip of the Rush nail was exposed through the fracture site. The humeral head was well below the level of glenoid, close to the thoracic wall (Fig. 1).

He was taken to the operation theater after informed consent for open reduction and internal fixation using locked plate and sutures. The patient was positioned in a beach chair manner. A deltoid splitting approach was used. The fracture was exposed. Sutures were taken in the greater tuberosity fragment. The head of humerus 

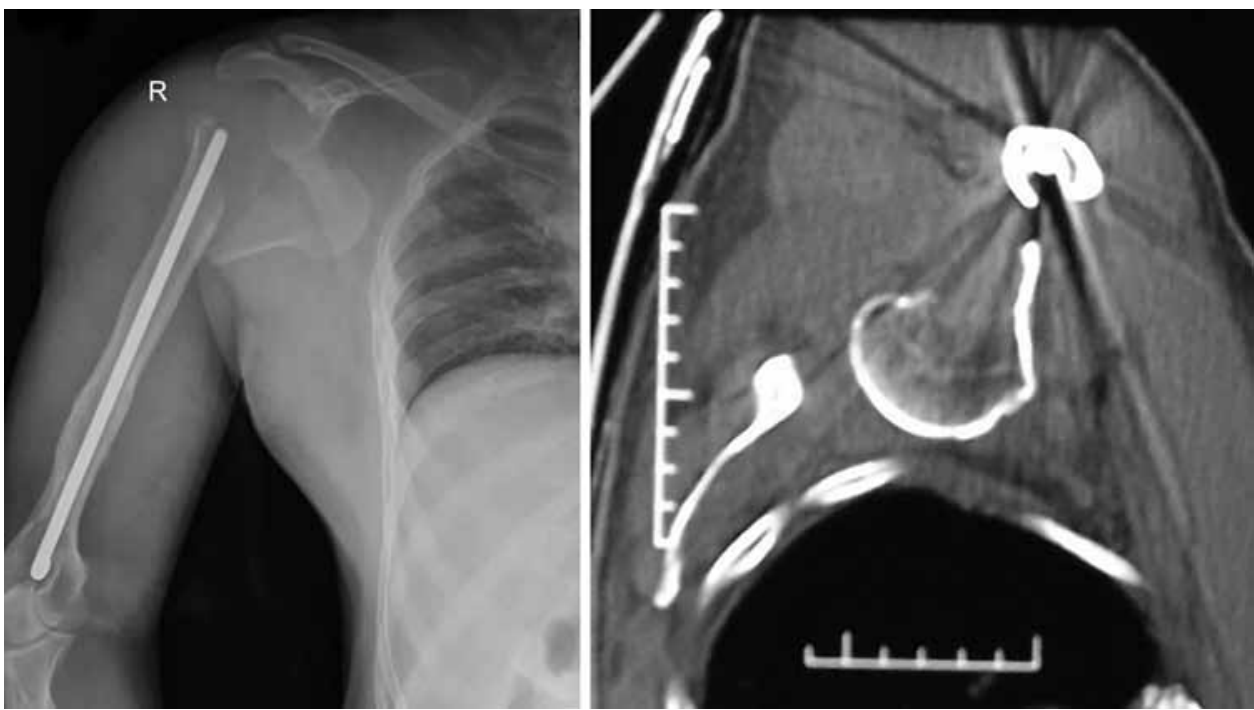

Fig. 1: Preoperative radiograph and axial cut of computed tomography scan showing proximity of humeral head to thoracic wall

had button-holed through the subscapularis muscle and was lying deep in the axillary pouch. A coracoid osteotomy was performed for adequate exposure. The head was lying just anterior to the neurovascular bundle, close to the thoracic cage. The head was pried out using bone lever. The Rush nail was extracted using a posterior approach in lower arm. The head was fixed to the shaft by a lag screw method. The greater tuberosity was reduced onto the head. The metaphyseal defect in the greater tuberosity was now seen which was filled with autogenous iliac crest cancellous bone graft. Stabilization was achieved using locked proximal humeral plate (PHILOS, Synthes). The rotator cuff was repaired. Wound was closed in layers over drain.

In the immediate postoperative period, the patient was given an abduction splint. Passive exercises were started as per the available ROM determined intraoperatively. Graduated physiotherapy was started subsequently under strict supervision. Radiographs at 1 year (Fig. 2) show satisfactory progress. There is no evidence of avascular necrosis of the humeral head. The patient has

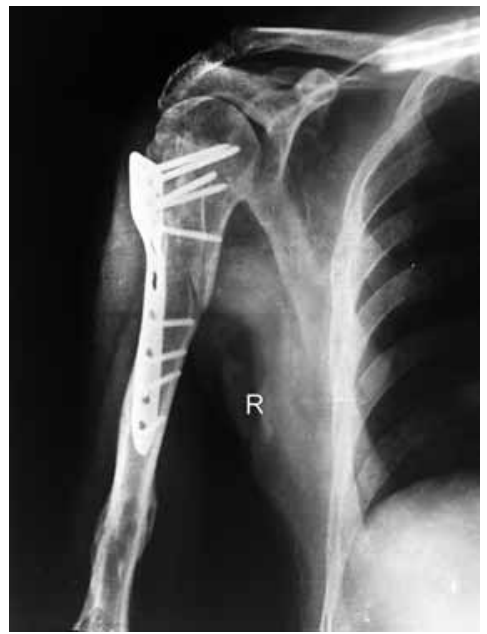

Fig. 2: Radiograph at 1 year follow-up terminal restriction of motion. He can comb his hair, lift overhead objects and dress on his own. He has mild pain on usual day to day activities.

\section{DISCUSSION}

Proximal humeral fractures account for about $3 \%$ of upper limb injuries. Three and four part fracture dislocation are relatively uncommon. In three and four part fractures of the proximal humerus the shaft is dissociated from the head at the level of the surgical or the anatomical neck. Our patient had an unusual fracture pattern. There was a long spiral fracture involving the proximal shaft of humerus. There have been reports of three-part fracture dislocations with ipsilateral shaft humerus fracture. ${ }^{2}$ However, to the best of the authors knowledge, there are no reports of three-part fractures where the fracture involves the proximal shaft instead of the surgical/anatomical neck.

The mechanism of injury in the three-part fracture dislocations usually is due to fall on an outstretched arm as was seen in this patient. However, due to the presence of the Rush nail there was an unusual fracture pattern. We believe that the proximal tip of the Rush nail acted as stress riser leading to a fracture around the tip of the nail. The stress concentration around the tip of an intramedullary nail is well known in lower limbs. However, its occurrence in upper limb is not well described. Our patient suffered a high velocity road traffic accident where he was violently thrown off his motorcycle due to collision with a car. The energy generated by this impact was sufficient to dislocate the glenohumeral joint fracturing the greater tuberosity. During the recoil, due to anatomical block, the fracture of the proximal shaft of humerus took place at the site of maximum stress concentration, in this case, at the tip of the Rush nail. Literature on fractures about an intramedullary nail is sparse. McKee et $\mathrm{al}^{3}$ 
described three cases of fractures at the distal end of locking humerus nail. Their cases involved fractures occurring in the early postoperative period. All the fractures in their series occurred at the site of distal locking screw.

In a fracture dislocation of proximal humerus, the position of the dislocated head usually subcoracoid. In our case, the humeral head was abutting the thoracic rib cage. This finding prompted us to thoroughly evaluate the patient for associated chest injuries. Fortunately the patient did not suffer any thoracic injuries. Migration of the fractured head into the thoracic cavity has also been reported. ${ }^{4}$ The degree of trauma along with other factors decide the amount of displacement. The migration of the dislocated head close to the thoracic wall required an extended approach by an osteotomy of the coracoid.

\section{CONCLUSION}

Occurrence of dissociation of the head of humerus from the shaft below the level of surgical neck has been reported here. The tip of a pre-existing intramedullary implant can act as a stress concentration point causing fracture at the level of tip instead of the usual level of the surgical neck. Displacement of the humeral head close to the thoracic wall should prompt thorough evaluation for chest injuries and requires an extended approach for retrieval and fixation.

\section{REFERENCES}

1. Neer CS 2nd. Displaced proximal humeral fractures: part I. Classification and evaluation. JBJS Am 1970;52-A(6):1077-1089.

2. Maffulli N, Yip KM, Cowman JE, Chan KM. Fracture of the shaft of the humerus with a fracture-dislocation of the same shoulder: a case report. Int Orthop 1996;20(4):237-238.

3. McKee MD, Pedlow FX, Cheney PJ, Schemitsch EH. Fractures below the end of locking humeral nails: a report of three cases. J Orthop Trauma 1996;10(7):500-504.

4. Daffner SD, Cipolle MD, Phillips TG. Fracture of the humeral neck with intrathoracic dislocation of the humeral head. J Emerg Med 2010;38(4):439-443. 